\title{
Acceso y permanencia de estudiantes de educación superior en la ciudad de Pilar
}

\author{
Iván Jorge Luis Acuña Brítez \\ acunaivan.py@gmail.com \\ Facultad de Humanidades y Ciencias de la Educación \\ Universidad Nacional de Pilar
}

\section{RESUMEN}

En el Paraguay se ha logrado importantes avances en cuanto a las oportunidades de acceso en los últimos años. Las universidades son instituciones que deben responder a los cambios y problemas sociales e ir evolucionando para dar respuestas a las necesidades de la comunidad. El acceso a la educación superior en el Paraguay, no es un privilegio de todos, aunque en los últimos tiempos hubo un gran aumento en la creación de universidades, todavía un gran porcentaje de la población paraguaya no tiene acceso a la educación superior. Asimismo, las instituciones de educación superior actualmente se enfrentan problemas como la deserción estudiantil, y deben responder a nuevos retos que plantean una sociedad en constante evolución. El objetivo general es fue determinar el acceso y permanencia de estudiantes de educación superior en la ciudad de Pilar. La investigación se enmarca en el enfoque cualitativo, pues se recolectan datos para describir el fenómeno estudiado a partir de las experiencias de los participantes. La investigación se enmarca en un diseño no experimental; los datos se recolectan sin la manipulación deliberada e intencional de las variables, se realiza tal y como se da en el contexto. La deserción universitaria en el Paraguay está asociada a problemas económicos y familiares, bajo rendimiento académico, poca motivación, elección incorrecta de la carrera y el trabajo; considerando que en el país la mayoría de los estudiantes no se dedican a tiempo completo al estudio y a la vez realizan algún tipo de labor.

Palabra clave: acceso; alumnos; permanencia; universidades. 


\title{
Access and permanence of higher education students in the city of Pilar
}

\begin{abstract}
In Paraguay, significant progress has been made in terms of access opportunities in recent years. Universities are institutions that must respond to social changes and problems and evolve to respond to the needs of the community. Access to higher education in Paraguay is not everyone's privilege, although in recent times there has been a great increase in the creation of universities, a large percentage of the Paraguayan population still does not have access to higher education. Likewise, higher education institutions currently face problems such as student dropout, and must respond to new challenges posed by a society in constant evolution. The general objective was to determine the access and permanence of higher education students in the city of Pilar. The research is framed in the qualitative approach, since data is collected to describe the phenomenon studied from the experiences of the participants. The research is framed in a non-experimental design; the data is collected without deliberate and intentional manipulation of the variables; it is done as it occurs in the context. University desertion in Paraguay is associated with economic and family problems, low academic performance, little motivation, incorrect choice of career and work; considering that in the country most of the students do not dedicate themselves full time to study and at the same time carry out some type of work.
\end{abstract}

Keyword: access; students; permanence; universities.

Artículo recibido: 15 octubre. 2021 Aceptado para publicación: 18 noviembre 2021 Correspondencia: acunaivan.py@gmail.com Conflictos de Interés: Ninguna que declarar 


\section{INTRODUCCIÓN}

En el Paraguay se ha logrado importantes avances en cuanto a las oportunidades de acceso en los últimos años. Aunque se habilitaron varias universidades sin el debido control, muchas de estas instituciones ofrecen la oportunidad de acceder a una educación superior a pobladores de zonas alejadas de los principales centros urbanos (Cultura., 2012)

Las universidades son instituciones que deben responder a los cambios y problemas sociales e ir evolucionando para dar respuestas a las necesidades de la comunidad, es por ello que esta investigación se plantea como objetivo general conocer los retos de las universidades en el Paraguay del siglo XXI.

Desde su creación definitiva en el año 1994 la Universidad Nacional de Pilar con sede central en la ciudad de Pilar, en la actualidad es una de las mejores del Paraguay buscando la excelencia académica en sus diferentes facultades, acompañados por sus docentes calificados que favorece el desarrollo educativo.

Otro problema que se percibe en el país es la deserción o no culminación de la educación media de parte de muchos jóvenes, lo cual imposibilita cursar un estudio universitario. Por lo tanto, para mejorar el acceso a la educación superior, se requiere un esfuerzo desde los niveles inferiores.

\section{El acceso a las Universidades}

\subsection{Sistemas de acceso a las universidades}

En el Paraguay básicamente existen tres formas de acceder a las universidades, que dependen de la naturaleza de las instituciones. La primera es la realización de un curso probatorio de ingreso de carácter eliminatorio, donde los estudiantes que logran los mejores puntajes acceden a la universidad y aquellos que logran los puntajes más bajos quedan afuera. Este sistema es utilizado en las universidades nacionales, donde el cupo para ingresar es limitado considerando que el presupuesto de la institución es del Estado. No obstante, en algunas instituciones privadas también se aplica este sistema en carrera como Medicina que exige una buena preparación de parte de los postulantes (CARRO, 2015)

Al respecto, entre los objetivos del Curso Probatorio de Ingreso de la carrera de Ingeniería Agronómica y afines de la Universidad Nacional de Asunción se menciona: “Lograr una selección más objetiva, posibilitando el ingreso de aquellos estudiantes con 
mayor aptitud y vocación para las Carreras ofrecidas" (GESTOSO \& Nidia Glavinich, 2016)

\subsection{Oportunidades de acceso a las universidades}

En cuanto a las oportunidades de acceso últimamente se ha logrado importantes avances, en cierto sentido se ha democratizado más la educación superior. Hasta 1990 solo existían dos universidades que se concentraban en Asunción y algunas ciudades importantes del interior del país, esto implicaba para los estudiantes abandonar su ciudad natal en muchos casos para cursar la universidad. Con el objetivo de dar oportunidad de acceso, en los últimos años se crearon varias universidades sin el debido de control, lo cual ha ido en detrimento de la calidad de los servicios educativos que se ofrecen.

No obstante, aún es limitado el acceso a la educación superior para los sectores más vulnerables que constituyen la población de escasos recursos del Paraguay. Según los datos de la Dirección General de Encuestas Estadísticas y Censos (2018) el 20\% de la población paraguaya sobrevive en la extrema pobreza con un ingreso mensual inferior al 500.000 guaraníes. Esto implica no contar con los recursos suficientes para afrontar una educación universitaria.

\subsection{La oferta de universidades y carreras}

Según los datos presentados por el Ministerio de Educación y Cultura sobre educación superior (2012, Pág. 4) en el Paraguay existen actualmente52 Universidades; que desarrollan como mínimo tres áreas del saber, de las cuales 8 son oficiales y 44 privadas, con 290facultadesque ofertan 1945 carreras.

En el mismo informe se menciona que se llegó a tener hasta 54 universidades, de las cuales una no está en funcionamiento y dos se fusionaron. (Cultura., 2012)

Desde 1991 comenzó la creación de universidades un poco después de la caída de la Dictadura Stronista. Ante esta situación, los expertos en educación superior consideran que existen necesidades como de contar con un marco normativo, tener claridad respecto a la situación de la educación superior, poner en orden la educación superior y que las universidades respondan a los problemas emergentes.

\subsection{La demanda de acceso a las Universidades}

Basualdo (2005, Pág.10) cita un informe del Consejo Nacional de Educación y Cultura donde se sostiene que en las universidades públicas, la mayor concentración de 
estudiantes se encuentra en las facultades de Ciencias Económicas y de Derecho, este hecho se atribuye al bajo costo de las carreras, el horario nocturno y las posibilidades de inserción laboral.

Según el informe publicado por el Viceministerio de Educación Superior en el Paraguay, actualmente hay unos 300.000 matriculados en universidades e institutos, lo cual ni siquiera alcanza el cinco por ciento de las personas que debería estar cursando la educación terciaria.

Por otra parte, de acuerdo con los datos presentados por el Viceministerio de la Juventud, los jóvenes paraguayos no se encuentran ante un panorama fácil, aunque la misma conforme más del $27 \%$ de la población nacional, históricamente ha encontrado escaso apoyo del Estado en materias importantes, tales como: educación, trabajo, salud, cultura y participación. (Basualdo Navarro, 2011)

El promedio de jóvenes no sobrepasa los 9 años de educación, y pensar en asistir a la universidad es, para la absoluta mayoría, poco menos que una utopía. El desempleo golpea fuerte a un $16 \%$ de los jóvenes, los más afectados por esta situación, y el $80 \%$ de los mismos carece de seguro médico.

\subsection{Circunstancias asociadas al acceso}

Para facilitar u ofrecer oportunidades de acceso a las universidades, existen organismos que otorgan becas a estudiantes de escasos recursos y con buen rendimiento académico. Entre estos organismos figuran la Itaipu, el Ministerio de Educación y Cultura, la Secretaría Técnica de Planificación, algunas Embajadas, las Gobernaciones, y las mismas universidades que tienen también sus programas de becas.

En cuanto a las becas que fueron otorgadas por Itaipu en desde el 2011 hasta ahora, año del Bicentenario de la independencia nacional, uno de los requisitos fue ser egresado de la Educación Media de alguna institución de gestión oficial. Se priorizaron las carreras que van a tener impacto en las necesidades de la población paraguaya. En este sentido, prevalecieron las carreras relacionas a Ciencias de la Salud, Ciencias de la Ingeniería y Ciencias Agropecuarias.

Además de los requisitos mencionados los postulantes debieron contar con promedio de calificación final en la Educación Media igual o mayor a la nota (cuatro), en una escala de calificaciones del uno al cinco o su equivalente; pertenecer a familias de escasos recursos económicos, interés en las carreras universitarias correspondientes a la presente 
convocatoria, aprobar las pruebas de capacidades elementales de Lengua Castellana y de Matemática, conforme a los requerimientos establecidos en el Reglamento y, estar dispuesto a trasladarse para residir en el lugar donde funciona la Universidad asignada, si fuere necesario.

\section{MARCO LEGAL}

\subsection{Constitución Nacional del Paraguay (Paraguay, 1992)}

\section{Capítulo VII - De la educación y de la cultura}

\section{Artículo 73 - Del derecho a la educación y de sus fines}

Toda persona tiene derecho a la educación integral y permanente, que como sistema y proceso se realiza en el contexto de la cultura de la comunidad. Sus fines son el desarrollo pleno de la personalidad humana y la promoción de la libertad y la paz, la justicia social, la solidaridad, la cooperación y la integración de los pueblos; el respeto a los derechos humanos y los principios democráticos; la afirmación del compromiso con la Patria, de la identidad cultural y la formación intelectual, moral y cívica, así como la eliminación de los contenidos educativos de carácter discriminatorio.

La erradicación del analfabetismo y la capacitación para el trabajo son objetivos permanentes del sistema educativo.

\section{Artículo 74 - Del derecho de aprender y de la libertad de enseñar}

Se garantizan el derecho de aprender y la igualdad de oportunidades al acceso a los beneficios de la cultura humanística, de la ciencia y de la tecnología, sin discriminación alguna. Se garantiza igualmente la libertad de enseñar, sin más requisitos que la idoneidad y la integridad ética, así como el derecho a la educación religiosa y al pluralismo ideológico.

\section{Artículo 75 - De la responsabilidad educativa}

La educación es responsabilidad de la sociedad y recae en particular en la familia, en el Municipio y en el Estado.

El Estado promoverá programas de complemento nutricional y suministro de útiles escolares para los alumnos de escasos recursos.

\section{Artículo 76 - De las obligaciones del estado}

La educación escolar básica es obligatoria. En las escuelas públicas tendrá carácter gratuito. El Estado fomentará la enseñanza media, técnica, agropecuaria, industrial y la superior o universitaria, así como la investigación científica y tecnológica. 
La organización del sistema educativo es responsabilidad esencial del Estado, con la participación de las distintas comunidades educativas. Este sistema abarcará a los sectores públicos y privados, así como al ámbito escolar y extraescolar.

\section{Artículo 77 - De la enseñanza en lengua materna}

La enseñanza en los comienzos del proceso escolar se realizará en la lengua oficial materna del educando. Se instruirá asimismo en el conocimiento y en el empleo de ambos idiomas oficiales de la República

En el caso de las minorías étnicas cuya lengua materna no sea el guaraní, se podrá elegir uno de los dos idiomas oficiales.

\section{Artículo 78 - De la educación técnica}

El Estado fomentará la capacitación para el trabajo por medio de la enseñanza técnica, a fin de formar los recursos humanos requeridos para el desarrollo nacional.

\section{Artículo 79 - De las universidades e institutos superiores}

La finalidad principal de las universidades y de los institutos superiores será la formación profesional superior, la investigación científica y la tecnológica, así como la extensión universitaria.

Las universidades son autónomas. Establecerán sus estatutos y formas de gobierno y elaborarán sus planes de estudio de acuerdo con la política educativa y los planes de desarrollo nacional. Se garantiza la libertad de enseñanza y la de la cátedra. Las universidades, tanto públicas como privadas, serán creadas por ley, la cual determinará las profesiones que necesiten títulos universitarios para su ejercicio.

\subsection{Ley $\mathrm{N}^{\circ} 1264$ General de Educación del Paraguay (EDUCACIÓN, 1998) SECCIÓN VI: Educación Superior}

Artículo 47.- La educación superior se ordenará por la ley de educación superior y se desarrollará a través de universidades e institutos superiores y otras instituciones de formación profesional del tercer nivel.

Artículo 48.- Son universidades las instituciones de educación superior que abarcan una multiplicidad de áreas específicas del saber en el cumplimiento de su misión de investigación, enseñanza, formación y capacitación profesional y servicio a la comunidad. 
Artículo 49.- Son institutos superiores, las instituciones que se desempeñan en un campo específico del saber en cumplimiento de su misión de investigación, formación profesional y servicio a la comunidad.

Artículo 50.- Son Instituciones de formación profesional del tercer nivel, aquellos institutos técnicos que brindan formación profesional y reconversión permanente en las diferentes áreas del saber técnico y práctico, habilitando para el ejercicio de una profesión. Serán autorizadas por le M. E. C. El título de técnico superior permitirá el acceso al ejercicio de la profesión y a los estudios universitarios o a los proveídos por los institutos superiores, que se determinen, teniendo en cuenta las áreas de su formación académica.

Artículo 51.- Las instituciones de formación del $3^{\circ}$ nivel el M. E. C. deberá priorizar los institutos de formación docente formarán para:

a. capacitar a los educadores con la más alta calidad profesional, científica y ética;

b. lograr el eficaz desempeño de su profesión en cada nivel del sistema educacional y diversas modalidades de la actividad educativa;

c. actualizar y perfeccionar permanentemente a los docentes en ejercicio; y,

d. fortalecer su competencia en el campo de la investigación educativa y desarrollo de la teoría y práctica de ciencias de la educación.

Artículo 52.- El ejercicio de la profesión docente se regirá por las normas de esta ley y por el Estatuto del Personal de la Educación.

Artículo 53.- Las universidades públicas y privadas e instituciones superiores de enseñanza, son parte del sistema nacional de educación. Su funcionamiento se adecuará a la legislación pertinente. El Consejo Nacional de Educación y Cultura evaluará periódicamente su funcionamiento y elevará el correspondiente informe al Congreso Nacional para su oportuna consideración.

\subsection{Ley $N^{\circ}$ 4.995/13 Educación Superior (SUPERIOR, 13)}

Capitulo IV - de los Cursos de Pre-Grado, las Carreras de Grado y los Programas de Postgrado de la Educación Superior

Artículo 61.- La educación superior desarrollará cursos de pre-grado, carreras de grado y programas de postgrado. 
Capítulo I de los cursos De Pre-Grado, Carreras de Grado y Programas de Postgrado

\section{SECCIÓN I}

\section{De los Cursos de Pre-Grado}

Artículo 62.- Los cursos de pre-grado están orientados a:

a. la preparación para ocupaciones de carácter operativo e instrumental para el ejercicio de una profesión técnica. Otorga el título de Técnico Superior en una y especialidad técnica específica.

b. la formación inicial de profesionales de la educación para el desempeño de la docencia en cada uno de los niveles del sistema educacional en las diversas modalidades de la actividad educativa. Otorga el título de Profesor.

c. le formación de profesionales de la educación para el desempeño de la función técnica en diversas áreas. Otorga el título de Técnico Docente en un área específica.

\section{SECCIÓN II}

\section{De las Carreras de Grado}

Artículo 63.- Las carreras de grado tendrán una duración mínima de 4 (cuatro) años y 2700 (dos mil setecientas) horas cursadas.

De acuerdo con los estándares internacionales vigentes, las carreras podrán tener una duración de 5 (cinco) a 6 (seis) años. Estas carreras otorgan el título correspondiente a una profesión o a los conocimientos académicos de una disciplina.

\section{JUSTIFICACIÓN}

Las universidades son instituciones de educación superior que evolucionan constantemente conforme la sociedad va cambiando y presentando nuevas exigencias. El Paraguay no está ajeno a problemas que demandan respuestas de las instituciones de educación superior. Esta investigación es de gran importancia por que aborda los desafíos o retos de las universidades en el Paraguay del siglo XXI, describe los aspectos que deben mejorar, cuáles son sus debilidades y fortalezas. Ofrece una visión general de lo que es la educación en este nivel.

El acceso a la educación superior en el Paraguay, no es un privilegio de todos, aunque en los últimos tiempos hubo un gran aumento en la creación de universidades, todavía un gran porcentaje de la población paraguaya no tiene acceso a la educación superior. Asimismo, las instituciones de educación superior actualmente se enfrentan problemas 
como la deserción estudiantil, y deben responder a nuevos retos que plantean una sociedad en constante evolución. (Basualdo Navarro, 2011)

En el Paraguay existen escasos datos estadísticos sistematizados sobre la deserción universitaria, lo que dificulta la obtención de resultados generales o nacionales sobre el problema

En una investigación realizada en la Universidad Católica Unidad Académica de Carapeguá (2009) que incluyó a cinco carreras de la cohorte de ingreso de los años 2012 y 2013a los cuales se les hizo un seguimiento de 5 años hasta la graduación, se detectó que la deserción afecta a 100 estudiantes de un total de 258 alumnos, lo cual constituye el 39\% del total. Así también se descubrió que el abandono de la carrera afecta ligeramente en mayor cantidad a los varones en comparación con las mujeres. Estos datos pueden servir como referencia en cuanto a la deserción en las universidades de carácter privado.

El problema que implica la deserción estudiantil en las universidades exige a las instituciones la ejecución de medidas para la retención de alumnos. Algunos de los mecanismos utilizados para retener estudiantes son por ejemplo la implementación de tutorías y clases de refuerzo, la concesión de becas y créditos educativos, un sistema de evaluación de varias oportunidades, la flexibilización del currículum, etc.

\section{OBJETIVOS DE LA PROPUESTA}

\subsection{Objetivo General}

- Determinar el acceso y permanencia de estudiantes de educación superior en la ciudad de Pilar

\subsection{Objetivos Específicos}

- Describir los sistemas y las oportunidades de acceso a la educación superior en la ciudad de Pilar.

- Explicar las políticas sobre la permanecía de los estudiantes en la educación superior en la ciudad de Pilar.

- Analizar los desafíos con respecto al acceso, permanencia de estudiantes.

\section{Diseño de las Actividades}

\section{Enfoque de la investigación}

La investigación se enmarca en el enfoque cualitativo, pues se recolectan datos para describir el fenómeno estudiado a partir de las experiencias de los participantes. 
(Hernández Sampieri, 2014)

Relacionado a ello, Hernández Sampieri (2014), expresa que: “el enfoque cualitativo consiste en obtener las perspectivas y puntos de vista de los participantes.

\section{Diseño de la investigación}

La investigación se enmarca en un diseño no experimental; los datos se recolectan sin la manipulación deliberada e intencional de las variables, se realiza tal y como se da en el contexto. De carácter fenomenológico pues el estudio busca comprender los aspectos relacionados al estrés a partir de la perspectiva de cada uno de los involucrados. (Hernández Sampieri, 2014)

Así también el autor mencionado con anterioridad expresa que: "el carácter fenomenológico tiene como objetivo principal explorar, describir y comprender las experiencias de las personas con respecto a un fenómeno y descubrir los elementos en común de tales vivencias”. (Hernández Sampieri, 2014).

\section{Alcance de la investigación}

La investigación es descriptiva. Se describen y detallan las características más importantes del fenómeno estudiado.

Hernández Sampieri (2014), al respecto, menciona que; “con los estudios descriptivos se busca especificar propiedades y características importantes de cualquier fenómeno que se analice".

\section{CONCLUSIONES}

Los sistemas de admisión que se aplican en las universidades estatales son el curso probatorio de carácter eliminatorio y el curso de admisión no eliminatorios cuyo objetivo es dotar a los estudiantes de competencias generales para afrontar la universidad. En cambio, las universidades privadas implementan el curso de admisión no eliminatorio y el acceso directo.

Los organismos o instituciones encargadas de ofrecer becas y ayudas a los estudiantes son la Itaipu, el Ministerio de Educación y Cultura, las embajadas extranjeras como las de Venezuela, Cuba, entre otras; la Secretaría Técnica de Planificación. Para acceder a las becas los requisitos principales son el buen rendimiento académico y pertenecer a familias de escasos recursos.

La deserción universitaria en el Paraguay está asociada a problemas económicos y familiares, bajo rendimiento académico, poca motivación, elección incorrecta de la 
carrera y el trabajo; considerando que en el país la mayoría de los estudiantes no se dedican a tiempo completo al estudio y a la vez realizan algún tipo de labor.

Las universidades, específicamente las privadas, para contrarrestar la deserción estudiantil ofrecen becas, descuentos en las cuotas, implementan clases de refuerzos, tutorías y en sus reglamentos establecen un sistema de evaluación con más de una oportunidad, para que los estudiantes tengan mayores posibilidades para aprobar la materia.

\section{REFERENCIAS BIBLIOGRAFICAS}

Basualdo Navarro, M. B. (2011). Deserción y repitencia en la educaciónsuperior universitaria en Paraguay. Asunción-Paraguay: El lector.

Cabero Almenara, J. (2016). Sobre la aceleración cultural. Sevilla: Renacimiento.

Carro, P. (2015). El trabajo es una de las principales causas de la deserción universitaria. Asunción : El lector.

Castro, C. d. (2004). La era de la información . Washington: Chrysalis.

Cultura., P. M. (2012). Datos sobre la Educación Superior en Paraguay. Asunción: Santillana.

Educación, L. N. (1998). Sección VI: Educación Superior. Asuncion: El Lector.

Fernández, R. (2016 ). Las Nuevas Tecnologías Aplicadas a la Educación. Madrid: MCGrawHill.

Gestoso, C. G., \& Nidia Glavinich, Z. G. (2016). Educación superior y empleo en Paraguay. . Asunción: AZETA S.A.

Gómez M., R. S. (2015). El uso académico de las redes sociales en universitarios. Educomunicación, 38.

Hernández Sampieri, R. \&. (2014). Metodología de la Investigación. Madrid: MCGrawHill. .

Martínez Sánchez, F. P. (2010). Nuevas Tecnologías . Mexico: MCGrawHill.

Moreira, M. A. (2009). La reconceptualización de la Tecnología Educativa desde unIntroducción a la Tecnología Educativa. Madrid: MCGrawHill.

Moreira, M. Á. (2018). Introducción a la Tecnología Educativa. «La reconceptualización de la Tecnología Educativa desde una multidisciplinar y crítica de las ciencias sociales»., 20.

Paraguay, C. N. (1992). Capitulo VII. Art 73 - 77. Asunción : ABC. 
Pérez, C. A. (2013). La tecnología educativa en la era de la información. Mexico: BUAP.

Robinson, M. (2014). Using e-mail and the Internet in science teaching. 11. Robinson, M. (2014) Using e-mailJournal of Information Technology for Teacher Education., 229-238.

Robles, A. y. (12 de 02 de 2013). Entornos virtuales de aprendizaje. Recuperado el 15 de 02 de 2020, de Nuevos retos educativos. : www.researchgate.net/profil

Salinas, J. (2014). Innovación docente y uso de las TIC en la enseñanza universitaria. Madrid: McGraw-Hill Interamericana .

SUPERIOR, L. N. (13). Titulo IV. Asunción: El lector.

Van Arken, H. (2018). Modelos pedagógicos de la escuela tradicional. Tecnologia Educativa, 33-36.

Zapata, M. (2017). Educación a distancia y educación cooperativa. Medios y Educación, $57-9$. 